\title{
MOSTRA DE SABERES DA EDUCAÇÃO PROFISSONAL EM SAÚDE DA ESCOLA DE SAÚDE PÚBLICA DO CEARÁ: COMPARTILHANDO APRENDIZAGENS
}

\author{
P. E. SILVA* e F. J. F. MOREIRA \\ Escola de Saúde Pública do Ceará \\ patyelizabeth@gmail.com*
}

Artigo submetido em outubro/2015 e aceito em dezembro/2015

DOI: $10.15628 /$ rbept.2015.3501

\section{RESUMO}

A Escola de Saúde Pública do Ceará (ESP/CE) tem o compromisso de formação e valorização dos profissionais de saúde do Estado. Seu núcleo da educação profissional atualmente desenvolve onze cursos de formação técnica, destinado aos profissionais que já atuam nos serviços públicos. Os alunos dos cursos técnicos em saúde em execução participam da Mostra de Saberes da Educação Profissional, realizada desde 2012, envolvendo também os gestores das instituições de saúde, docentes e colaboradores da Escola. Este estudo tem como objetivo descrever essa Mostra. Seu propósito é valorizar a formação técnica no Ceará, e dar visibilidade aos processos formativos e de qualificação da força de trabalho de nível médio em saúde. Sua realização oportuniza o aprimoramento da educação profissional em saúde e o compartilhamento de processos de aprendizagem. Essa estratégia adotada pela ESP/CE possibilita a visibilidade do ensino profissional oferecido, demonstrando através dos trabalhos desenvolvidos pelos alunos, o conjunto determinado de saberes construído, contribuindo para o ensino profissionalizante no Ceará.

PALAVRAS-CHAVE: Educação em Saúde, Educação Profissional em Saúde Pública.

\section{KNOWLEDGE OF HEALTH VOCATIONAL EDUCATION FAIR OF PUBLIC HEALTHCARE SCHOOL OF CEARÁ: SHARING THE LEARNING}

\begin{abstract}
The Public Healthcare School of Ceará (ESP / EC) is committed to the training and development of the state's health professionals. The core of education is currently conducting eleven technical training courses targeted to professionals already engaged in public services. Students of current health technical courses participate in the Knowledge of Healthcare Vocational Education Fair, held since 2012, involving the managers of healthcare institutions, teachers and employees of the school. This study aims to describe this fair. Its purpose is
\end{abstract}

to enhance technical training in Ceará, and give visibility to the training and qualification of the average level of the workforce in healthcare processes. Its realization favors the improvement of professional education in healthcare and learning processes sharing. This strategy adopted by ESP / EC enables the visibility of vocational education offered, demonstrating through the work developed by the students, the particular set of knowledge built, contributing to the vocational education in Ceará.

KEYWORDS: Healthcare Education, Public Healthcare Education Professional. 


\section{INTRODUÇÃO}

O acesso à educação tanto geral quanto profissional não foi ofertado a milhares de brasileiros ao longo do tempo, o que na impossibilidade de conclusão dos estudos, acarretou na inserção no mercado de trabalho em saúde como forma de subsistência. Nesse contexto, os trabalhadores que atuaram e atuam na área da saúde sem formação profissional e qualificação específica para o trabalho que desenvolvem, não alcançam crescimento profissional, nem melhorias de salário, ou registro profissional nos conselhos de classe. Dessa forma, esses trabalhadores têm sido excluídos, com pouco reconhecimento profissional e social (SÓRIO, 2002).

A história da educação profissional em saúde expõe que as políticas de formação dos trabalhadores em saúde progrediram sob a referência da integração da formação dos trabalhadores com a realidade dos serviços. O objetivo principal aparece nas diferentes políticas como: o Projeto Larga Escala, nos anos 1980; o Programa de Profissionalização dos Auxiliares de Enfermagem (PROFAE), nos anos 1990; e a Educação Permanente em Saúde, nos anos 2000, como a transformação de práticas na perspectiva da atenção integral à saúde (RAMOS, 2009).

Diante da grande importância dos profissionais de nível médio na área da saúde para o Sistema Único de Saúde (SUS), visto que estes realizam atividades vitais para o bom funcionamento do serviço, o que se percebe é que eles ainda passam por um processo de invisibilidade como resultado de um modelo que está centrado na figura do médico, assim como pela própria divisão social do trabalho em saúde, configurado pela fragmentação no processo do cuidar e a separação entre teoria e prática ou concepção e execução (GALVÃO, 2013).

A gestão eficiente do SUS visa uma política de valorização dos profissionais de saúde, considerando-se que há uma precarização dos vínculos e falta de perspectivas de carreira, assim como baixa remuneração, condições ruins de segurança ocupacional e falta de representação coletiva.

Vários fatores como a exclusão do processo educativo; o déficit de escolas profissionais para atender às necessidades de qualificação de pessoal para a área da saúde, na quantidade e qualidade necessárias; a falta de uma política e de artifícios que possibilitassem a formação em saúde; os diferentes perfis sociais dos trabalhadores já empregados; e as qualificações iniciais feitas por meio de treinamentos pontuais realizados em serviço, justificam a necessidade de criação de escolas específicas para formação dos trabalhadores da saúde, na tentativa de oportunizar a estes, ensino de qualidade (SÓRIO, 2002).

Desta forma, considerando a necessidade de fortalecimento da educação profissional em saúde, tendo em vista o atendimento das demandas do SUS, as Escolas Técnicas de Saúde do SUS (ETSUS), no Brasil, foram criadas em sua maioria na década de 80 num contexto de redemocratização da sociedade brasileira. Surgiram como estratégia frente ao problema da baixa qualificação da força de trabalho empregada nos serviços de saúde, e tinham como principal missão "promover a profissionalização dos trabalhadores de nível médio, sem qualificação específica para o desenvolvimento das ações de saúde" (SÓRIO; LAMARCA, 1998, p. 149).

A Escola de Saúde Pública do Ceará (ESP/CE) é uma autarquia vinculada à Secretaria da Saúde do Estado do Ceará (SESA-CE), para operacionalizar a política de desenvolvimento de pessoas para o SUS e compor uma rede de formação, em parceria com universidades, escolas, cursos profissionalizantes, favorecendo uma unidade na diversidade de pensamento sobre o 
profissional necessário para responder às necessidades da população e, portanto, do sistema de saúde.

Seu núcleo da Educação Profissional tem por missão

\begin{abstract}
"Contribuir para o fortalecimento do SUS, por meio do desenvolvimento de programas de qualificação, educação profissional técnica e educação permanente do(a)s trabalhadore(a)s no nível da educação básica, bem como do desenvolvimento de pesquisa e extensão, a partir das necessidades sociais e do SUS e da construção de redes colaborativas" (CEARÁ, 2012).
\end{abstract}

A ESP/CE, através da Dieps, realiza a Mostra de Saberes da Educação Profissional, envolvendo alunos dos cursos técnicos em execução, gestores das instituições de saúde, docentes e colaboradores da Escola. Essa atividade surgiu por se considerar que a aprendizagem é um processo de aprimoramento de habilidades e mudança de atitudes, porque há um novo ambiente de aprendizagem, permeado pela participação, mediação e interatividade dos envolvidos, levando o sujeito ao desenvolvimento de competências.

Diante desse contexto, o objetivo deste texto é promover uma reflexão sobre a visibilidade e valorização da educação profissional em saúde, trazendo como estratégia, a Mostra de Saberes da Educação Profissional, desenvolvida pela Diretoria de Educação Profissional em Saúde (Dieps) da Escola de Saúde Pública do Ceará (ESP/CE), que tem como propósito valorizar a formação profissional técnica no Ceará, e dar visibilidade aos processos formativos e de qualificação da força de trabalho de nível médio em saúde, com os quais alunos e docentes das Escolas são protagonistas dos processos emancipadores e transformadores das práticas, voltadas aos cuidados prestados no âmbito do SUS.

\title{
2 EDUCAÇÃO PROFISSIONAL EM SAÚDE
}

O ensino profissionalizante no Brasil nasceu sob o estigma da discriminação, e até hoje persiste este estigma de que o ensino profissionalizante é para aqueles das classes menos favorecidas, enquanto "o saber" é para a elite. A história do ensino brasileiro mostra essa dualidade e reforça a estigmatização do processo educacional do trabalhador (NASCIMENTO; COLLARES, 2005).

O período de 1970 foi marcado pela Lei $n \circ$ 5.692/71, que reformulou o ensino de 1 ㅇ e 2은 graus no país, estabelecendo compulsoriamente a profissionalização como única finalidade para o ensino de $2 \circ$ grau (CAMPELO; LIMA FILHO, 2009). A Lei citada reconheceu a integração completa entre o ensino profissionalizante e o sistema regular de ensino, rompendo com uma tradição secular que não vinculava o ensino médio exclusivamente ao ensino profissionalizante, e tornou obrigatório que o estudante adquirisse uma profissão, mesmo aquele que buscava o $2 \circ$ grau como meio de alcançar o ensino superior (NASCIMENTO; COLLARES, 2005).

A reforma da educação profissional ocorreu em consequência do Decreto 2.208/97 e do Programa de Expansão da Educação Profissional (PROEP) e suas ações decorrentes. Dessa forma o ensino médio retomou um sentido unicamente propedêutico em termos legais, enquanto os cursos técnicos, separados do ensino médio obrigatoriamente, passaram a ser oferecidos de duas 
formas: concomitante ao ensino médio, mas com matrículas e currículos distintos; ou subsequente ao ensino médio (PACHECO, 2012).

O dualismo entre a formação geral e formação profissional foi reforçado com a aplicação desse decreto, característico da educação profissional brasileira, mantendo em sua estrutura a separação entre a formação geral, destinada ao preparo para o ensino superior, e a formação técnica, que preparava para o mercado de trabalho (COELHO, 2013).

Ao se fazer a opção pela escola unicamente profissionalizante para os trabalhadores técnicos em saúde, a maioria deles com escolaridade básica incompleta, acabou-se corroborando o princípio da dualidade educacional brasileira, ou seja, educação básica de qualidade para a elite, e escola profissionalizante para os trabalhadores (RAMOS, 2009).

As atuais exigências de qualificação profissional dos trabalhadores apontam para um perfil diferenciado dos profissionais, mais amplo em termos de competências, mais polivalente em termos de atuação e baseado em princípios concretos de conhecimentos. Não se tratando apenas de treinamento e apreensão de habilidades técnicas, mas de construção de capacidade.

Para superar o problema da invisibilidade e desvalorização dos profissionais de nível técnico da saúde faz-se necessário o estabelecimento de políticas que levem a decisões colegiadas, trabalho em equipe e valorização dos servidores, referente tanto aos salários quanto aos processos de educação permanente, o planejamento participativo, a autonomia e a responsabilização das tarefas a eles confiadas, além de um processo de avaliação dos serviços e trabalhadores, redundando em motivação financeira e de mérito (GALVÃO, 2013).

\section{ESCOLAS TÉCNICAS DE SAÚDE DO SUS}

As ETSUS são espaços estratégicos de formação e qualificação para o SUS e têm como finalidade promover a profissionalização dos trabalhadores da saúde inseridos nos serviços de saúde, procurando formá-los de acordo com os novos modelos assistenciais. À medida que aumenta o compromisso do gestor com a saúde pública e o entendimento de que uma assistência de qualidade está diretamente relacionada a uma melhor formação dos trabalhadores que ali atuam, há o fortalecimento das ETSUS e de seus propósitos (PEREIRA; RAMOS, 2006).

Essas Escolas se propuseram a proporcionar aos trabalhadores o conhecimento sob sua prática de trabalho e a vinculá-la aos princípios do SUS, que se pautam no conceito ampliado de saúde. Porém, os fundamentos epistemológicos e éticos-políticos que orientaram suas concepções e práticas, têm-se colocado como limites ao enfrentamento da dualidade educacional brasileira (RAMOS, 2009).

Sob a dimensão ético-política, cabem as ETSUS: nortear as políticas públicas de formação profissional de pessoal de nível médio para a saúde; ordenar, trabalhar e buscar caminhos para a formação de trabalhadores no campo da educação profissional de nível médio em saúde; dar visibilidade às políticas de educação profissional em saúde; incluir trabalhadores nos processos educativos de forma ordenada e sistemática; certificar os trabalhadores nos campos educacional e laboral; e fortalecer o potencial da Rede de Escolas Técnicas do SUS (RET-SUS) como instâncias certificadoras da educação profissional de nível médio em saúde (GALVÃO, 2009). 
As ETSUS se diferenciam das demais instituições de ensino profissionalizante por serem descentralizadas e terem como foco principal o aluno-trabalhador. Fortalecer as ETSUS é fazer com que se possa enfrentar o desafio de universalizar a formação técnica profissional e a educação permanente para o SUS.

A ESP/CE compõe a RET-SUS e direciona seus programas educacionais para profissionais já inseridos nos serviços de saúde. Sua atuação tem como referência a Política Nacional de Educação Permanente em Saúde, bem como as necessidades de formação dos profissionais de nível médio no âmbito do Estado do Ceará, desenvolvendo projetos e programas na área de educação profissional técnica, pós-técnica, de educação permanente, pesquisa e extensão. Nesta perspectiva, a ESP/CE, através da Dieps, apresenta um portfólio de 16 cursos, dentre livres (básico, atualização e aperfeiçoamento), técnicos e pós-técnicos que fazem parte do planejamento de sua oferta atual e para os próximos anos.

\section{MOSTRA DE SABERES COMO ESTRATÉGIA PARA VISIBILIDADE E VALORIZAÇÃO DA EDUCAÇÃO PROFISSIONAL EM SAÚDE}

Desde o ano de 2012, a ESP/CE através da Dieps, realiza a Mostra de Saberes da Educação Profissional da ESP/CE - Compartilhando aprendizagens. Seus objetivos são: compartilhar aprendizagens entre alunos, educadores, trabalhadores da ESP/CE e os gestores dos serviços de saúde; demonstrar o conhecimento adquirido ao longo do curso, através de trabalhos realizados na área da formação técnica para saúde; valorizar a formação profissional técnica na saúde; e dar visibilidade à educação profissional, oportunizando a manifestação das competências desenvolvidas, durante o ano, em cada um dos cursos.

Esse evento vem reforçar o propósito da Educação Profissional da ESP/CE: uma educação voltada para a aprendizagem aplicada à realidade, promovendo uma significação do conhecimento, aliado às práticas profissionais, visando ao fortalecimento da atuação do profissional de saúde. Trata-se de uma atividade que valoriza a formação técnica, tornando-se a cada ano, uma atividade que oportuniza o compartilhamento de processos de aprendizagem e o aprimoramento da educação profissional em saúde, contribuindo para a produção do conhecimento.

A I Mostra de Saberes da Educação Profissional em Saúde da ESP/CE foi realizada em novembro de 2012, e contou com a participação dos cursos técnicos em Análises Clínicas, Radiologia, Citopatologia e Hemoterapia. Foi dividida em dois momentos: o primeiro, realizado no auditório da ESP/CE, com a apresentação do perfil das turmas, das profissões envolvidas, onde foram convidados profissionais de cada área/curso participante para falar sobre as especificidades do profissional que recebe a formação técnica, e uma mesa redonda com a discussão sobre essas profissões. No segundo momento, houve a exposição de 14 trabalhos desenvolvidos pelos alunos dos cursos técnicos participantes, referentes ao conteúdo específico daquela formação, estudado naquele ano.

A II Mostra de Saberes aconteceu em outubro de 2013, desta vez com um diferencial, os melhores trabalhos apresentados foram selecionados para serem expostos na $V$ Exposição da Escola de Saúde Pública do Ceará (EXPOESP), outra importante atividade da ESP/CE, que aconteceu dentro do XIII Congresso das Secretarias Municipais de Saúde do Ceará. 
Participaram da II Mostra de Saberes, os alunos dos cursos técnicos em Análises Clínicas, Citopatologia, Enfermagem, Hemoterapia, Radiologia, Saúde Bucal e Vigilância em Saúde. Foram selecionados 12 trabalhos a serem expostos na V EXPOESP realizada em novembro de 2013 no Centro de Eventos do Ceará.

Em outubro de 2014, aconteceu a III Mostra de Saberes envolvendo os alunos/profissionais dos sete cursos técnicos em andamento no período: Agente Comunitário de Saúde, Apoio ao Acolhimento em Saúde, Atendimento pré-hospitalar, Enfermagem, Prótese Dentária, Saúde Bucal, Vigilância em Saúde e do Projeto Caminhos do Cuidado. Os trabalhos apresentados abordaram diversos assuntos tratados em sala de aula e passaram por avaliações realizadas pelos coordenadores e supervisores pedagógicos dos cursos técnicos da Dieps, através de um instrumento elaborado pela coordenação pedagógica da Escola. Ao todo, foram desenvolvidos e apresentados pelos alunos, 31 trabalhos.

A participação do Projeto Caminhos do Cuidado, nessa Mostra se deu com a apresentação do trabalho sobre a formação em saúde mental (crack, álcool e outras drogas) para os Agentes Comunitários de Saúde, auxiliares e técnicos em enfermagem da atenção básica.

A Mostra de Saberes é uma estratégia da ESP/CE que propõe dar visibilidade e valorização da educação profissional em saúde, ao oportunizar aos alunos dos cursos técnicos mostrarem, através de trabalhos, aos docentes, gestores dos serviços de saúde e demais alunos dos cursos técnicos diferentes dos seus, o conhecimento adquirido durante o curso, demonstrando o que foi significativo nesse aprender, assim como para a Escola, que terá uma devolutiva do trabalho que vem executando junto a esses alunos.

\section{CONSIDERAÇÕES FINAIS}

Novos enfoques teóricos e de produção tecnológica no campo da saúde passaram a exigir novos perfis profissionais. Por isso, tornou-se imprescindível e obrigatório o comprometimento das instituições formadoras com o SUS. As instituições formadoras e as de saúde possuem a condição de reconstituírem a si mesmas, pois tradicionalmente as primeiras são o campo de exercício do ensino, e as outras, o campo de exercício dos serviços. Quanto maior o comprometimento dessas instâncias, maior a probabilidade de mudarem a si mesmas.

Cabe ao SUS e às instituições formadoras coletar, sistematizar, analisar e interpretar permanentemente informações da realidade, problematizar o trabalho e as organizações de saúde e de ensino, e construir significados e práticas com orientação social, mediante participação ativa dos gestores, formadores, usuários e estudantes.

O funcionamento de um sistema de saúde não se resume à atuação do médico e de outros profissionais de nível superior. $\mathrm{O}$ trabalho do profissional técnico em saúde vem sendo visto como um trabalho complementar, subordinado aos profissionais de nível superior, com pouca ou nenhuma autonomia, o que reflete na invisibilidade da profissão.

Os trabalhadores de nível médio também têm um papel de grande importância nos serviços de saúde. Existe vários técnicos e auxiliares realizando atividades vitais para o bom funcionamento do serviço, o que enfatiza a importância da formação e qualificação dos trabalhadores de nível médio para o SUS. 
É necessário também dar visibilidade ao ensino profissionalizante, para isso, buscam-se entre outras coisas, escolas formadoras que não se limitem ao interesse imediato, pragmático e utilitário. Por essa concepção, o ensino profissionalizante deverá se estruturar em consonância com o avanço do conhecimento científico e tecnológico, vinculando a ciência com a prática, e superando a dicotomia entre formação teórica geral e técnica instrumental.

A visibilidade do ensino profissionalizante implica na articulação de competências com evidências a nível técnico, científico e relacional. A relevância profissional constrói-se a partir das atitudes individuais que formam o coletivo e que, por sua vez, se refletem na ampliação das intervenções sociais, mais expressivamente, na ocupação de espaços que deem margem e reconhecimento ao ensino técnico como protagonista de um importante saber e fazer.

Por sua vez, os profissionais técnicos podem contribuir para a valorização da profissão no intuito de construir, coletivamente, uma profissão mais influente, comprometida e participativa nas decisões de políticas públicas, sociais e institucionais. Devem buscar construir uma profissão mais atuante, que tenha o compromisso social necessário para conquistar maior autonomia, possibilitando espaços que deem ênfase à profissão, garantindo assim, a chance de construir uma nova imagem da educação profissional que reflita o seu verdadeiro potencial perante a sociedade. Este é o desafio também para as instituições formadoras, e no caso do SUS, das Escolas Técnicas.

A estratégia adotada pela ESP/CE de realizar a Mostra de Saberes da Educação Profissional possibilitou a visibilidade do ensino profissional oferecido pela Escola, demonstrando que através dos trabalhos desenvolvidos pelos alunos, um conjunto determinado de saberes foi construído, legitimando o conhecimento oferecido pela Escola, contribuindo para a formação dos alunos/trabalhadores e para o ensino profissionalizante no Ceará.

É necessário incentivar e despertar no alunos/profissionais dos cursos técnicos, a prática da pesquisa e realização de estudos e trabalhos que demonstrem a importância do trabalhador técnico para a saúde, o que poderá contribuir para um maior reconhecimento, valorização, melhora da autoestima destes, e maior visibilidade da profissão.

\section{REFERÊNCIAS}

1. CAMPELO, A. M. M. B.; LIMA FILHO, D. L. L. Educação profissional. In: PEREIRA, I. B.; LIMA, J. C. F (Org.). Dicionário da Educação Profissional em Saúde. Rio de Janeiro: EPSJV, 2009. 478p.

2. CEARÁ, ESCOLA DE SAÚDE PÚBLICA. Projeto político pedagógico. Fortaleza, 2012.

3. COELHO, J. E. O decreto no $2.208 / 97$ e a reforma da educação profissional na unidade Florianópolis da escola técnica federal de Santa Catarina. In: CONGRESSO BRASILEIRO DE HISTÓRIA DA EDUCAÇÃO, 7, 2013, Cuiabá. Anais dos Congressos Brasileiros de História da Educação. Cuiabá: CBHE, 2013. Disponível em: <http://sbhe.org.br/novo/congressos/cbhe7>. Acesso em: 25 out. 2014.

4. GALVÃO, E. A. A. Cenário nacional das escolas técnicas do SUS: a criação dos Cefor no Brasil. Saúde e Sociedade, v. 18, supl. 2, p. 60-63, 2009.

5. GALVÃO, E. A. A invisibilidade dos profissionais técnicos da saúde é real. Revista RET-SUS, ano VII, n. 61, p. 20-21, maio, 2013. Entrevista concedida a Jéssica Santos.

6. NASCIMENTO, M. I. M.; COLLARES, S. A. O. Análise da eficiência da Lei 5.692/71 na formação 
dos trabalhadores de Guarapuava sob a perspectiva de consciência para a cidadania e qualificação para o trabalho. Revista HISTEDBR [online], Campinas, n.20, p. 76 - 85, dez. 2005 - ISSN: 1676-2584.

7. PACHECO, E. (Org.) Perspectivas da educação profissional técnica de nível médio: propostas de diretrizes curriculares nacionais. São Paulo: Moderna, 2012.

8. PEREIRA, I. B.; RAMOS, M. N. Educação Profissional em Saúde. Coleção Temas em Saúde. Rio de Janeiro, Editora Fiocruz, 118p, 2006.

9. RAMOS, M. N. Concepções e práticas pedagógicas nas Escolas Técnicas do Sistema Único de Saúde: fundamentos e contradições. Trabalho, Educação e Saúde. Rio de Janeiro, v. 7, suplemento, p. 153-173, 2009.

10. SÓRIO, R. E. R. Educação profissional em saúde no Brasil: a proposta das escolas técnicas de saúde do sistema único de saúde. In: Formação / Ministério da Saúde. Projeto de Profissionalização dos Trabalhadores da Área de Enfermagem. - v. 2, n. 5, 2002. - Brasília: Ministério da Saúde, 2002.

11. SÓRIO, R. E. R.; LAMARCA, I. Novos desafios das escolas técnicas de saúde do SUS. Revista Saúde Coletiva, Rio de Janeiro, v. 8, n. 2, p. 147-164, 1998. 